\title{
Studies of Mira and Semiregular Variables Using Visual Databases
}

\author{
T. R. Bedding, B. C. Conn
}

School of Physics, University of Sydney 2006, Australia

\author{
A. A. Zijlstra \\ UMIST, Department of Physics, Manchester, UK
}

\begin{abstract}
We used wavelets to investigate period and amplitude changes in Mira and semiregular variables and found a variety of behaviours. Period and amplitude changes often go together, perhaps because changes in amplitude are causing period changes via nonlinear effects.
\end{abstract}

Wavelet analysis is useful for tracing changes in the periods and amplitudes of long-period variables. We have already published results for the semiregular variable R Dor, which appears to switch between two modes on a time scale of decades (Bedding et al. 1998). See also the work of Szatmáry, Kiss and co-workers (Kiss et al. 1999, 2000), and of Andronov (2000).

In the case of Miras, a few stars are known to have long-term period trends that may be related to adjustment after a helium shell flash (Wood \& Zarro 1981). A good example is R Aql, which has a gradually decreasing period and, as is clear in Fig. 1, a previously unnoticed decrease in amplitude. We find a similar effect in other Miras known to have changing periods, such as W Dra and $\mathrm{BH}$ Cru (periods and amplitudes both increasing) and R Hya and T UMi (periods and amplitudes decreasing). The case of BH Cru is shown in Fig. 2.

Other stars such as S Ori (Fig. 3) show repeated period changes, but still with matching amplitude changes. We suggest that, at least in some cases, the amplitude changes might cause the period changes via nonlinear effects.

Acknowledgments. We thank the many observers and those who maintain the visual databases of the AAVSO, RASNZ, AFOEV, VSOLJ and BAAVSS.

\section{References}

Andronov, I. A. 2000, in these proceedings, p. 85

Bateson, F. M., McIntosh, R., \& Venimore, C. W. 1988, RASNZ PubVSS, 15, 70

Bedding, T. R., Zijlstra, A. A., Jones, A., \& Foster, G. 1998, MNRAS, 301, 1073

Foster, G. 1996, AJ, 112, 1709

Kiss, L. L., Szatmáry, K., Cadmus, Jr., R. R., \& Mattei, J. A. 1999, A\&A, 346, 542 
Kiss, L. L., Szabó, Gy., Szatmáry, K., \& Mattei, J. A. 2000, in these proceedings, p. 117

Percy, J. R. \& Au, W. W.-Y. 1999, PASP, 111, 98

Walker, W. S. G., Ives, F. V., \& Williams, H. O. 1995, Southern Stars, 36, 123

Whitelock, P. A. 1999, in Stellar Evolution on Human Time Scale, ed. E. F. Guinan \& R. H. Koch, New Astr. Rev., 43, 437 [astro-ph/9801002]

Wood, P. R. \& Zarro, D. M. 1981, ApJ, 247, 247

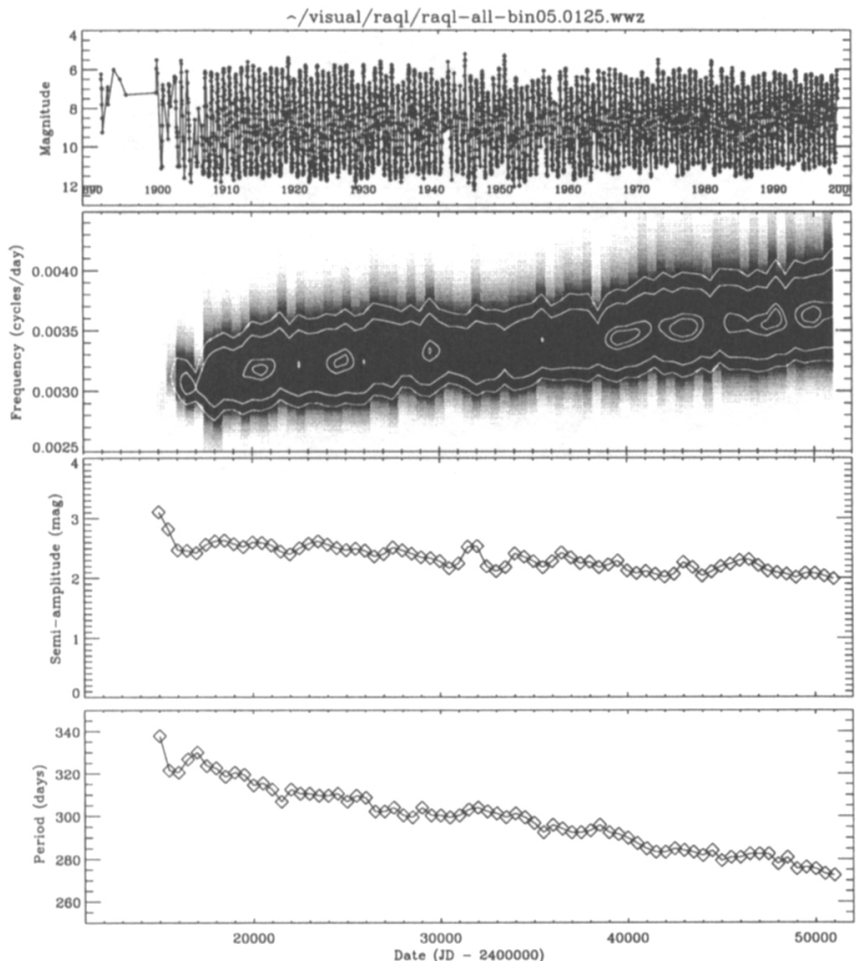

Figure 1. Analysis of visual observations of R Aql. The top panel shows the light curve averaged in 5-d bins, from data supplied by the AAVSO, AFOEV, VSOLJ and BAAVSS. The second panel shows the WWZ wavelet transform, while the third and fourth panels show time evolution of the semi-amplitude and period, respectively. As described by Bedding et al. (1998), we use the wavelet implementation by Foster (1996), with the semi-amplitude and period being derived from the WWA rather than the WWZ. 


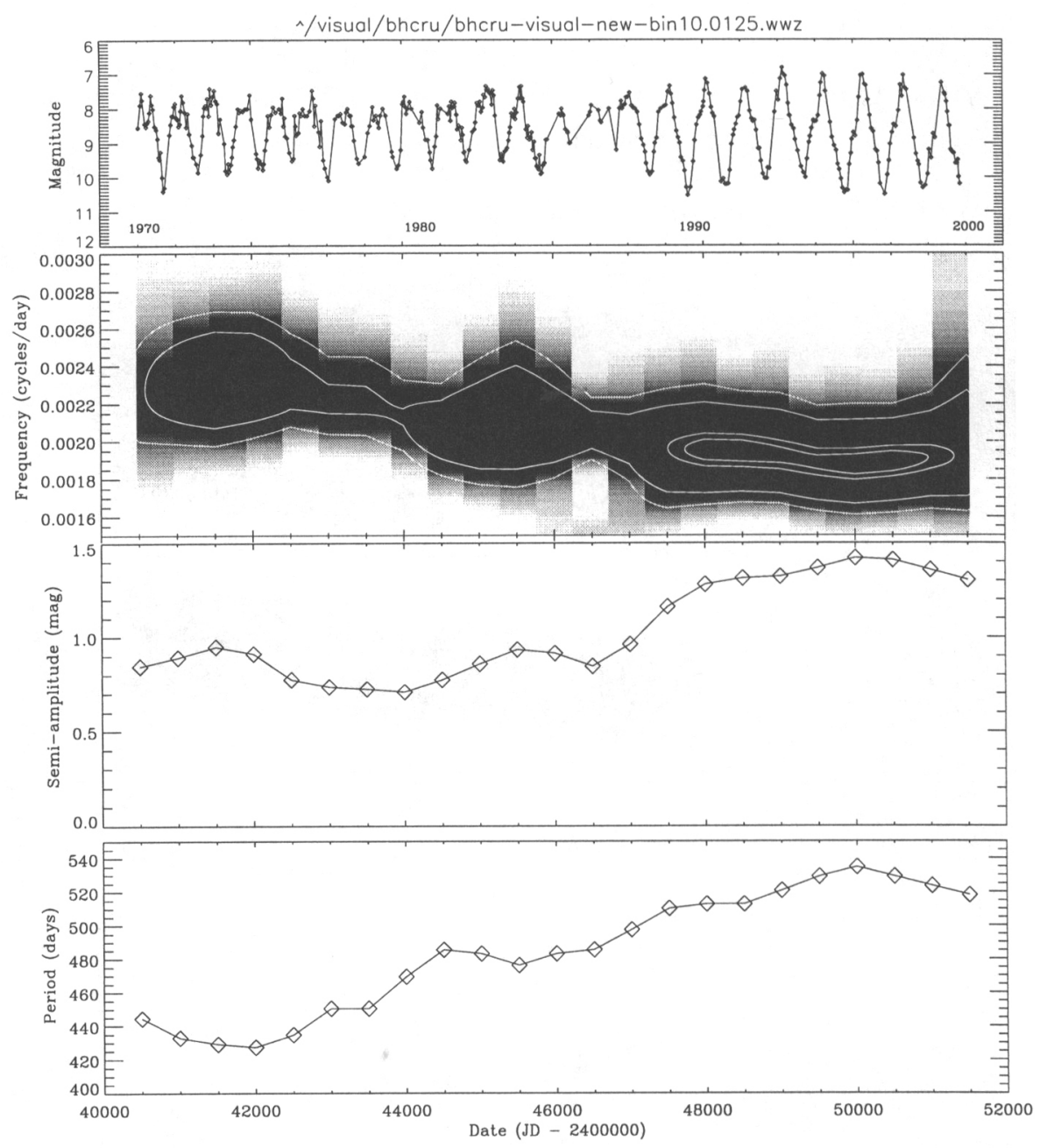

Figure 2. Same as Fig. 1 but for BH Cru, from data supplied by the RASNZ and averaged in 10-d bins. Our analysis confirms the suggestion by Bateson, McIntosh, \& Venimore (1988) that period of BH Cru is increasing. The rate of period change $\left(\dot{P}=0.012 \mathrm{~d} \mathrm{~d}^{-1}\right)$ is the largest we have found for any Mira. Meanwhile, as pointed out by Walker, Evans, \& Williams (1995) and confirmed by Hipparcos photometry, the double maxima in the light curve have now disappeared. As reviewed by Whitelock (1999), the spectrum of this star has changed from $\mathrm{O}$-rich to $\mathrm{C}$-rich, perhaps after dredge-up, and it is possible the period, amplitude and light curve changes are also related to a recent dredge-up event. 


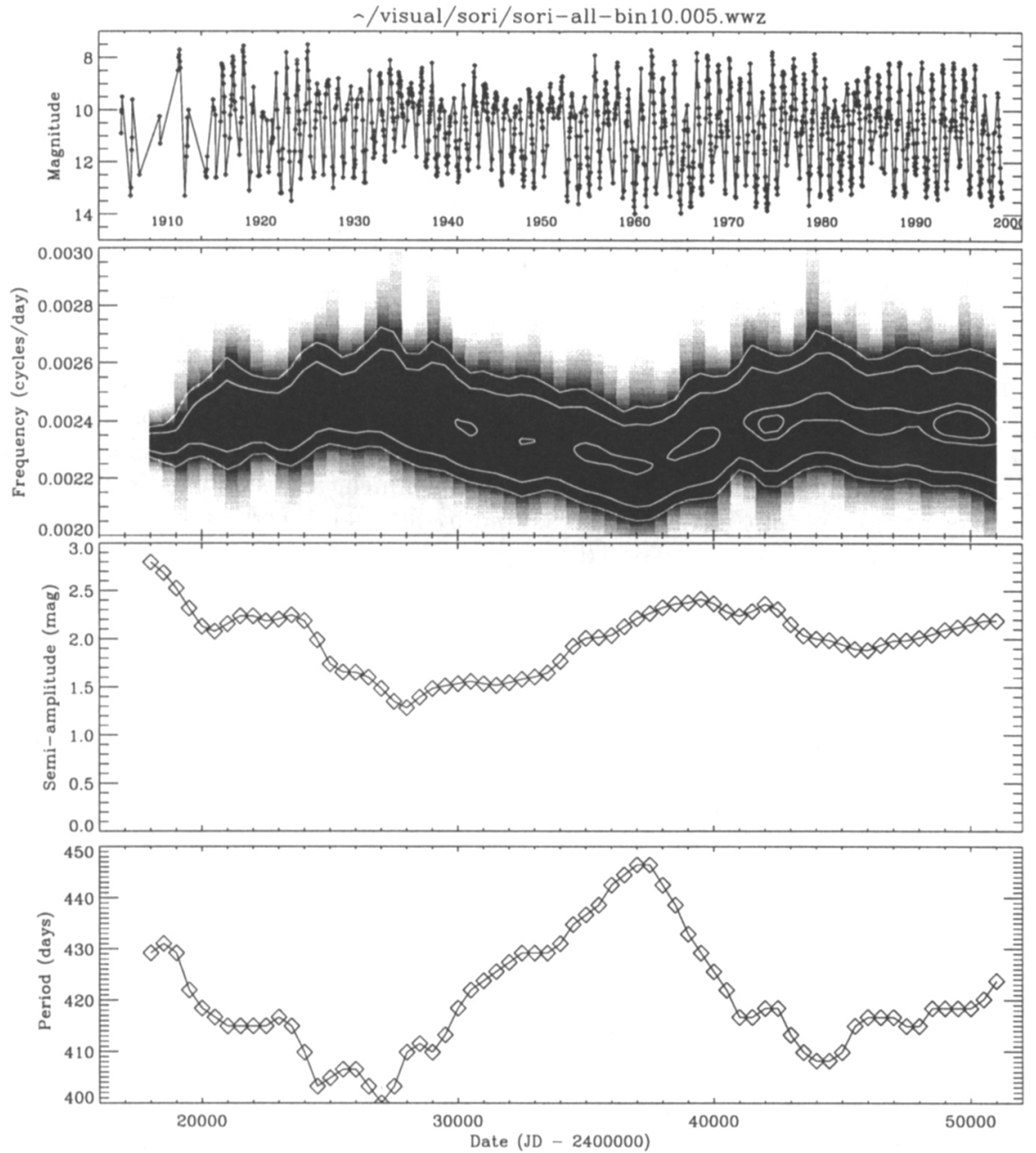

Figure 3. Same as Fig. 1 but for S Ori, from data supplied by the AAVSO, AFOEV and VSOLJ and averaged in 10-d bins. Period changes in this star are noted in the General Catalogue of Variable Stars and also by Percy \& Au (1999). Here, we see changes both in period and period derivative $(\dot{P})$. In fact, the period has not been constant at any time in the last 100 years, with $\dot{P}$ changing sign every few decades. Note that the true behaviour is difficult to recognise in the $O-C$ diagram, since a change in $\dot{P}$ must be deduced from a change in curvature. 


\section{Discussion}

Wojtek Dziembowski: Does R. Doradus remember pulsation phase when it returns to the previous mode?

Tim Bedding: No, it does not. It would be a bit surprising if it did, since LPVs generally show cycle-to-cycle fluctuations in their periods (they are not very stable in phase).

Frank Pijpers: To what extent is the radius of $\mathrm{R}$ Dor, as determined by interferometric techniques, model-atmosphere dependent (limb-brightening etc.)? (Is this insufficient for changing the identification from first overtone to fundamental?)

Tim Bedding: We also measured the angular diameter in the near infrared ( $J$ band), where limb-darkening and molecular absorption have much less effect on the angular diameter. It does not seem that incorrect modeling of these effects is enough to change the diameter by much - this also applies to other (genuine) Miras that have been measured by interferometry (e.g. Haniff, Scholz, \& Tuthill 1995, MNRAS 276,640).

Ivan Andronov: It seems that apparent parabola-like changes of the wavelet amplitude variations are caused by taking into account the parts of the light curve with different acting periods. Thus, the wavelet technique is useful for a preliminary analysis. Then it may be recommended to split the data into parts according to the acting period. In this case, the amplitude and phase variations with time will be less affected by the edge effect.

Géza Kovács: Nonlinear radial stellar pulsation models for Cepheids and R.R Lyrae stars show that the period increases with the amplitude of the limit cycle. This effect becomes stronger for more evolved objects such as W Vir stars. These results are in line with what you found observationally for semi-regular variables.

Robert Buchler: The mode-switching that you see in $R$ Doradus is quite similar to what a time-frequency analysis of R Scuti (an RV Tau-type star) displayed. In R Sct we could show that the irregular pulsations result from the nonlinear interaction of a linearly unstable mode with a linearly stable overtone that gets entrained through a near 2:1 resonance. 